\title{
Modelagem matemática do tempo de vida de baterias de Lítio Íon Polímero utilizando modelos elétricos combinados e genéricos
}

\author{
Marcia de Fatima Brondani Binelo ${ }^{1}$, Airam Teresa Zago Romcy Sausen ${ }^{1}$ and \\ Paulo Sérgio Sausen ${ }^{1}$
}

\author{
${ }^{1}$ Programa de Pós-Graduação em Modelagem Matemática (PPGMM), Unijuí, R. Lulu Ingelfritz, 480-Ijuí \\ (RS)-Brasil \\ *brondani.marcia@gmail.com; airam@unijui.edu.br; sausen@unijui.edu.br...
}

Submetido: 27/04/2018. Revisado: 14/10/2018. Aceito: 02/11/2018.

\section{Resumo}

A otimização do tempo de uso de dispositivos móveis depende da correta predição do tempo de vida da bateria. Neste trabalho é apresentada a análise do desempenho de dois modelos elétricos, o modelo combinado Chen e Rincón-Mora e o modelo genérico Battery, que são aplicados na modelagem matemática do tempo de vida de baterias de Lítio Íon Polímero. Os modelos são submetidos a um mesmo cenário de simulação, utilizando a mesma metodologia para a estimação dos seus parâmetros empíricos, que é baseada na análise visual de curvas de descarga obtidas experimentalmente. Para a validação dos modelos é considerado o mesmo conjunto de dados, composto de perfis de descarga constantes e variáveis, o que torna esse processo mais realista. Os experimentos são realizados em uma plataforma de testes, e os modelos são implementados na ferramenta computacional Matlab/Simulink (Student Suite). Os resultados, analisados a partir da comparação entre os tempos de vida simulados e os tempos de vida experimentais, mostram que ambos modelos são acurados, apresentando erros médios inferiores a $2 \%$. No entanto, o modelo Battery apresenta melhor desempenho médio e possuí um processo de parametrização mais simples quando comparado ao modelo Chen e Rincón-Mora.

Palavras-Chave: Estimação de parâmetros; modelo combinado; modelos elétricos; modelo genérico; tempo de vida da bateria

\begin{abstract}
The optimization of mobile devices use time depends on the correct prediction of the battery lifetime. This paper presents the performance evaluation of two electrical models, namely, the Chen and Rincón-Mora combined model, and the Battery generic model. These models are employed for the mathematical modeling of Lithium-ion Polymer batteries lifetime prediction. The models are submitted to the same simulation scenario, and the same methodology is used for the estimation of their empirical parameters, which is based on the visual analysis of experimentally obtained discharge curves. For the models validation, the same data set, composed of variable and constant discharge profiles, is used. The experiments are performed in a testbed, and the models are implemented using the Matlab/Simulink (Student Suite) tool. The comparison between the batteries lifetimes obtained by the models and those obtained experimentally show that the models are accurate, presenting mean errors below $2 \%$. However, the Battery model presents a better mean performance, and its parametrization process is simpler, when compared to the Chen and Rincón-Mora model parametrization.
\end{abstract}

Key words: Battery lifetime; combined model; electrical models; generic model; parameter estimation 


\section{Introdução}

O constante crescimento no desenvolvimento e no uso de tecnologias móveis, tais como dispositivos eletrônicos móveis e veículos elétricos ou híbridos que utilizam alguma forma de armazenamento de energia, tem gerado novos desafios, especialmente quanto à criação de novas tecnologias de baterias e questões associadas à sustentabilidade. Conforme dados publicados pela União Internacional de Telecomunicações, o número de assinaturas de telefones celulares aumentou de 5,29 bilhões em 2010 para aproximadamente 7,74 bilhões em 2017, o que equivale a 103,5 aparelhos para cada 100 habitantes do planeta (ITU; 2017). No setor automobilístico, segundo o relatório Global EV Outlook 2017, da Agência Internacional de Energia, o número estimado de veículos elétricos em circulação pode ser de até 70 milhões em 2025 (IEA; 2017). Diante deste cenário, existe atualmente uma crescente necessidade de pesquisas direcionadas para o desenvolvimento e evolução das baterias recarregáveis, que não estão conseguindo acompanhar, de maneira satisfatória e na mesma velocidade, os avanços tecnológicos, principalmente, no que diz respeito à capacidade nominal e à densidade de energia (Rezvanizaniani et al.; 2014). Em geral, essas limitações comprometem o tempo de vida da bateria, aqui entendido como o intervalo de tempo durante o qual a bateria, depois de totalmente carregada, pode fornecer energia ao aparelho antes de uma nova recarga ser necessária (Brondani et al.; 2017a).

Adiciona-se ainda a esta problemática, a importante questão ambiental do descarte das baterias não mais utilizadas em dispositivos eletrônicos e automóveis. Cabe reforçar que quanto menor for o tempo de vida de um bateria, e consequentemente, maior for o número de ciclos de recargas, mais cedo ela será substituida, e se descartada de forma indevida, irá aumentar, ainda mais, os vários problemas ambientais já enfrentados pelo planeta (Hammond and Hazeldine; 2015).

Neste contexto, muitos pesquisadores têm focado seus estudos na predição do tempo de vida de baterias a partir de modelos matemáticos (Doyle et al.; 1993; Suthar et al.; 2013; Chiasserini and Rao; 1999; Panigrahi T et al.; 2001; Rakhmatov and Vrudhula; 2001; Brand et al.; 2014; Chen and Rincón-Mora; 2006; Hu and Jung; 2013; Romio et al.; 2015). O tempo de vida da bateria é influenciado por dois importantes efeitos não lineares presentes no processo de descarga, o efeito de recuperação e o efeito taxa-capacidade (Rakhmatov and Vrudhula; 2001; Sausen et al.; 2010). Além disso, alguns eventos inevitáveis podem limitar o tempo de vida da bateria, tais como o modo como são realizadas a carga e a descarga, a quantidade de ciclos de uso, e o sistema de gerenciamento térmico e de energia (Manzetti and Mariasiu; 2015). Para enfrentar estes problemas, a modelagem matemática por meio de modelos matemáticos confiáveis, que sejam capazes de descrever de forma acurada o comportamento dinâmico da bateria sob diferentes condições de uso, passa a ter significativa importânica. Além disso, para muitas aplicações, é importante encontrar um equilíbrio entre a complexidade e a acurácia do modelo matemático utilizado, para que o mesmo possa ser facilmente embarcado em microprocessadores, fornecendo resultados precisos em tempo real (Pattipati et al.; 2011; Fotouhi et al.; 2016).

Dentre as classes de modelos matemáticos de bateria, denominadas conforme as abordagens de modelagem utilizadas, a classe dos modelos elétricos tem sido usada em uma ampla gama de aplicações, considerando diferentes tipos de baterias (Chen and Rincón-Mora; 2006; He et al.; 2012; Rezvanizaniani et al.; 2014; Luo et al.; 2016). Esses modelos fornecem informações importantes tais como corrente, tensão e estado de carga (SOC), podendo também incorporar alguns aspectos não lineares do problema, mantendo uma complexidade relativamente baixa ( $\mathrm{Hu}$ and Jung; 2013).

A classe dos modelos elétricos pode ser dividida em seis subclasses, as quais são: modelos Simples (Seaman et al.; 2014; He et al.; 2012), modelos baseados em Thévenin (He et al.; 2011; Lin et al.; 2016), modelos baseados em Impedância (Li et al.; 2014), modelos baseados em Runtime (Mousavi and Nikdel; 2014), modelos Combinados (Chen and Rincón-Mora; 2006; Zhang et al.; 2014) e modelos Genéricos (Tremblay et al.; 2007). Os modelos Simples representam a bateria por meio de uma resistência interna e de uma fonte de tensão ideal, comumente expressa pela tensão de circuito aberto (Voc) (He et al.; 2012). Os modelos da subclasse Thévenin fornecem as respostas transientes da bateria usando redes resistivas-capacitivas (RC) (Lin et al.; 2016), e são capazes de capturar dois efeitos dependentes do tempo, o efeito de depleção e o efeito de recuperação (Seaman et al.; 2014). Os modelos baseados em Impedância são fundamentados na técnica de Espectroscopia de Impedância Eletroquímica (EIE), em que cada componente dos circuitos está relacionado a um processo eletroquímico da bateria, e assim podem fornecer uma boa descrição do seu comportamento interno (Li et al.; 2014; Fotouhi et al.; 2016). Os modelos baseados em Runtime possibilitam simular o tempo de vida da bateria considerando descargas de correntes contínuas e variáveis, no entanto, não apresentam bons resultados para descargas de correntes variáveis devido à baixa precisão da modelagem das características transientes da bateria (Chen and Rincón-Mora; 2006; Mousavi and Nikdel; 2014). A subclasse de modelos Combinados consiste na combinação de diferentes modelos elétricos a fim de unir os melhores atributos de cada modelo, tais como, a correta predição do SOC e do tempo de vida da bateria, e as respostas em regimes estacionário e transiente (Zhang et al.; 2014; Chen and RincónMora; 2006). E, os modelos Genéricos descrevem o comportamento dinâmico de diferentes tecnologias de baterias sob diversas condições de carga e descarga (Tremblay et al.; 2007; Brondani et al.; 2017a). Uma revisão dos principais modelos que compõem cada uma dessas subclasses pode ser encontrada em Binelo et al. (2017).

Um modelo elétrico da subclasse de modelos Combinados, que tem sido aplicado para diferentes tecnologias de baterias, é o modelo proposto por Chen and Rincón-Mora (2006). Esse modelo, aqui denominado modelo Chen e Rincón-Mora, é 
considerado acurado na literatura e é capaz de capturar as características elétricas e dinâmicas da bateria, tais como, Voc, capacidade utilizável e resposta transiente. Na subclasse de modelos Genéricos, um modelo que se destaca é o modelo Battery. Esse modelo possui três sinais de saída: SOC, corrente de descarga e tensão, sendo capaz de simular o efeito de recuperação, que consiste na reorganização de elétrons no eletrólito durante intervalos de tempo em que a corrente de descarga é nula ou muito baixa (períodos de relaxamento) (Tremblay et al.; 2007).

Neste artigo é apresentada a análise do desempenho desses dois modelos elétricos, o modelo Combinado Chen e Rincón-Mora e o modelo Genérico Battery, quando aplicados à modelagem matemática do tempo de vida de baterias de Lítio Íon Polímero (LiPo), amplamente utilizadas em dispositivos móveis modernos e veículos elétricos, sendo também analisado o processo de parametrização dos modelos. Para fins de comparação, ambos modelos são submetidos a um mesmo cenário de simulação, que compreende a adoção de um mesmo método para a estimação dos seus parâmetros empíricos baseado na análise visual das curvas de descarga obtidas experimentalmente; assim como a utilização de um mesmo conjunto de dados experimentais, composto de perfis de descarga constantes e variáveis, para a validação dos referidos modelos. Os experimentos são realizados em uma plataforma de testes, e os modelos são implementados na ferramenta computacional Matlab/Simulink (Student Suite). Os resultados são analisados a partir da comparação entre os tempos de vida simulados e os tempos de vida experimentais. Pretende-se, desta forma, verificar qual desses dois modelos é o mais indicado, quanto à acurácia e ao processo de parametrização, para predizer o tempo de vida de baterias utilizadas em dispositivos móveis, veículos elétricos e híbridos.

$O$ restante deste artigo está organizado como segue. Na Seção 2 são apresentados os dois modelos elétricos aplicados à predição do tempo de vida de baterias, descrevendo suas equações e principais características. Na Seção 3 são apresentados os dados experimentais utilizados nos processos de parametrização e de validação dos modelos. Na Seção 4 são descritas as metodologias adotadas para a estimação dos parâmetros dos modelos. Na Seção 5 são apresentados os resultados das simulações computacionais e a análise comparativa do desempenho dos modelos. E, na Seção 6 é apresentada a conclusão.

\section{Modelos elétricos}

Nesta seção, inicialmente, é apresentado o modelo Chen e Rincón-Mora considerando as equações que descrevem o seu comportamento elétrico e os parâmetros que as compõem. Em seguida, é exposto o modelo Battery evidenciando suas particularidades quanto às baterias aqui estudadas.

\subsection{Modelo Chen e Rincón-Mora}

O modelo Chen e Rincón-Mora compreende dois circuitos, conforme mostrado na Figura 1, combinando características de modelos baseados em Runtime (circuito da esquerda) e em Thévenin (circuito da direita) (Chen and Rincón-Mora; 2006). $\mathrm{O}$ circuito da esquerda modela a capacidade, o SOC e o tempo de vida da bateria, e é composto por uma resistência de autodescarga $R_{a}$, uma capacitância $C_{3}$ e uma fonte de corrente controlada $i(t)$. O circuito da direita fornece a resposta transiente, sendo constituído por uma resistência $R_{0}$, duas redes RC dispostas em paralelo e uma fonte de tensão controlada $\operatorname{Voc}\left(V_{S O C}\right)$. A tensão $V_{S O C}$ representa o SOC da bateria e pode variar de $0 \mathrm{~V}$ (SOC igual a $0 \%$ ) a $1 \mathrm{~V}$ (SOC igual a 100\%) (Chen and Rincón-Mora; 2006).

A resposta transiente é modelada a partir de duas constantes de tempo, de curta e de longa duração, definidas no período de $t_{0}<t<t_{r}$, em que $t_{0}$ é o tempo inicial e $t_{r}$ é o tempo de término do período. Durante $o$ intervalo de $t_{0}<t<t_{d}$, a bateria é descarregada com uma corrente constante $(i(t)>0)$ e, de $t_{d}<t<t_{r}$, ela permanece ociosa $(i(t)=0)$, sendo $t_{d}$ o tempo final da descarga (Kim and Qiao; 2011). As resistências e capacitâncias $R_{1}, C_{1}, R_{2}$ e $C_{2}$ representam as respostas transientes de curta e de longa duração, respectivamente. Em estado estacionário, $C_{1}$ e $C_{2}$ desempenham a função de um circuito aberto, mas, em condição transiente, esses capacitores se comportam como um curto circuito para o momento transitório, até que estejam totalmente carregados (Chen and Rincón-Mora; 2006).

A capacitância $C_{3}$ representa a carga total armazenada na bateria e é obtida a partir de

$$
C_{3}=3600 \text { Capacityf } f_{1}(\text { Cycle }) f_{2}(\text { Temp }),
$$

onde: Capacity é a capacidade nominal (Ah), $f_{1}($ Cycle) é o fator de correção do número de ciclos e $f_{2}$ (Temp) é o fator de correção da temperatura (Chen and RincónMora; 2006).

Aplicando a lei das Malhas de Kirchhoff, tem-se a tensão de saída $V(t)$ expressa por

$$
V(t)=\operatorname{Voc}\left(V_{S O C}\right)-R_{0} i(t)-V_{\text {Transient }}(t),
$$

onde: $V_{\text {Transient }}(t)$ é a tensão transiente $(V)$, calculada a partir de

$$
V_{\text {Transient }}(t)=V_{1}+V_{2} \text {, }
$$

onde: $V_{1}$ é a tensão transiente de curta duração $(V)$ e $V_{2}$ é a tensão transiente de longa duração $(V)$, ambas dadas pelas equações (4) e (5), respectivamente,

$$
\begin{gathered}
V_{1}= \begin{cases}R_{1} i(t)\left[1-e^{-\frac{\left(t-t_{0}\right)}{\tau_{1}}}\right], & t_{0}<t<t_{d} \\
V_{1}\left(t_{d}\right) e^{-\frac{\left(t-t_{d}\right)}{\tau_{1}}}, & t_{d}<t<t_{r}\end{cases} \\
V_{2}= \begin{cases}R_{2} i(t)\left[1-e^{-\frac{\left(t-t_{0}\right)}{\tau_{2}}}\right], & t_{0}<t<t_{d} \\
V_{2}\left(t_{d}\right) e^{-\frac{\left(t-t_{d}\right)}{\tau_{2}}}, & t_{d}<t<t_{r}\end{cases}
\end{gathered}
$$

onde: $V_{1}\left(t_{d}\right)$ é a tensão transiente de curta duração no tempo final da descarga $(V), \tau_{1}=R_{1} C_{1}, V_{2}\left(t_{d}\right)$ é a tensão transiente de longa duração no tempo final da descarga $(V)$ e $\tau_{2}=R_{2} C_{2}$ (Kim and Qiao; 2011). 


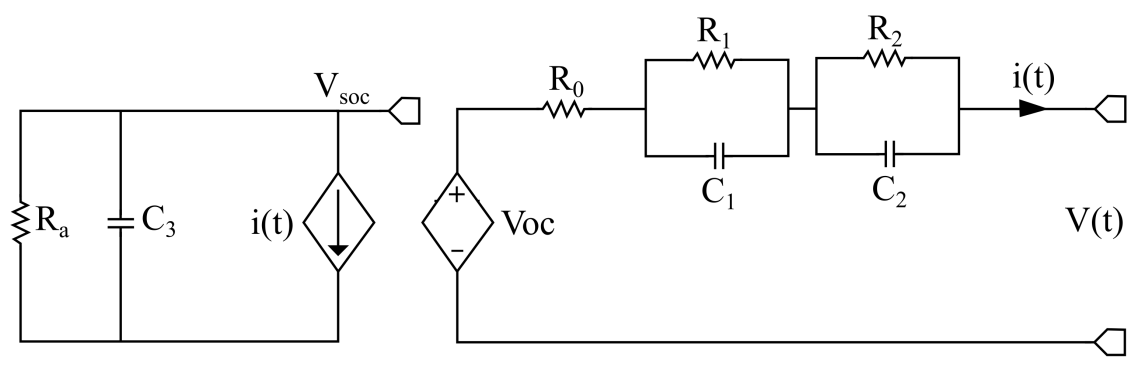

Figura 1: Circuito equivalente do modelo Chen e Rincón-Mora (Binelo et al.; 2017)

Os componentes Voc, $R_{0}, R_{1}, C_{1}, R_{2}$ e $C_{2}$, são funções de SOC, descritas pelas seguintes equações

$$
\begin{aligned}
& \operatorname{Voc}(S O C)=a_{0} e^{-a_{1} S O C}+a_{2}+ \\
& a_{3} S O C-a_{4} S_{O C}^{2}+a_{5} S C^{3} \text {, } \\
& R_{0}(S O C)=b_{0} e^{-b_{1} S O C}+b_{2} \text {, } \\
& \left\{\begin{array}{l}
R_{1}(S O C)=c_{0} e^{-c_{1} S O C}+c_{2}, \\
C_{1}(S O C)=d_{0} e^{-d_{1} S O C}+d_{2}, \\
R_{2}(S O C)=e_{0} e^{-e_{1} S O C}+e_{2}, \\
C_{2}(S O C)=f_{0} e^{-f_{1} S O C}+f_{2} .
\end{array}\right.
\end{aligned}
$$

A metodologia adotada na literatura para a estimação dos 21 parâmetros das equações (6), (7) e (8) é baseada na técnica de ajuste de curvas, utilizando quatro curvas pulsadas de descargas de baterias (Chen and Rincón-Mora; 2006).

\subsection{Modelo Battery}

O modelo Battery possibilita simular o comportamento dinâmico de baterias recarregáveis sob diferentes condições de uso (Tremblay et al.; 2007). A resistência interna é considerada constante e a capacidade efetiva não sofre alterações com a variação da amplitude da corrente, sendo negligenciados os efeitos da temperatura, da autodescarga e de memória (Tremblay and Dessaint; 2009). Este modelo é integrado à biblioteca SimPowerSystems, na ferramenta computacional Matlab/Simulink (MathWorks; 2017).

Os sinais de saída (SOC, corrente e tensão) referentes a processos de carga e descarga dependem do tipo de bateria a ser simulado pelo modelo. $\mathrm{Na}$ Figura 2 é apresentado o circuito equivalente do modelo Battery para o caso específico estudado neste trabalho, ou seja, descarga de baterias de LiPo. A tensão de saída, segundo o conceito da lei das Malhas de Kirchhoff, é dada por

$$
V_{s}(t)=f\left(i t, i^{*}, i, \operatorname{Exp}\right)-R i,
$$

onde: $R$ é a resistência interna $(\Omega), i$ é a corrente elétrica $(A)$, e $f\left(i t, i^{*}, i, \operatorname{Exp}\right)$ é a tensão de descarga definida pela função

$$
\begin{gathered}
f\left(i t, i^{*}, i, \operatorname{Exp}\right)=E_{0}-\underbrace{K\left(\frac{Q}{Q-i t}\right) i t}_{\text {Pol. Voltage }}- \\
\underbrace{K\left(\frac{Q}{Q-i t}\right)}_{\text {Pol. Resistance }} i^{*}+A e^{-B i t},
\end{gathered}
$$

onde: $E_{0}$ é a tensão constante $(V), K$ é a constante de polarização $(V / A h)$ ou a resistência de polarização $(\Omega), Q$ é a capacidade máxima da bateria $(A h)$, it é a capacidade extraída $(A h), i^{*}$ é a corrente dinâmica em baixa frequência $(A), A$ é a amplitude da zona exponencial $(V)$, e $B$ é o inverso da constante do tempo da zona exponencial $\left((A h)^{-1}\right)$.

Alguns desses parâmetros são obtidos a partir de valores medidos em curvas de descarga de baterias. Na Figura 3 é ilustrada uma curva característica de descarga que pode ser considerada pelo modelo Battery. Os pontos $P_{1}\left(Q_{E x p}, V_{E x p}\right)$ e $P_{2}\left(Q_{\text {Nom }}, V_{\text {Nom }}\right)$ correspondem aos términos das zonas exponencial e nominal, respectivamente.

Assim, a tensão constante $E_{0}$ é obtida a partir de

$$
E_{0}=V_{\text {Full }}+R i-A,
$$

onde: $V_{\text {Full }}$ é a tensão da bateria com carga completa $(V)$. No instante em que a descarga é inicializada, $i t=0$ e $i^{*}=0$ (Tremblay and Dessaint; 2009).

Ao considerar o ponto $P_{1}$ obtém-se os parâmetros $A$ e $B$ da equação (10), determinados por meio das equações (12) e (13), respectivamente,

$$
\begin{gathered}
A=V_{\text {Full }}-V_{\text {Exp }}, \\
B=\frac{3}{Q_{\text {Exp }}},
\end{gathered}
$$

onde: $V_{\text {Exp }}$ é a tensão no final da zona exponencial $(V)$ e $Q_{E x p}$ é a capacidade no final da zona exponencial (Ah) (Tremblay et al.; 2007). por

$O$ parâmetro $K$ é obtido a partir do ponto $P_{2}$, dado

$$
K=\left(\frac{E_{0}-V_{N o m}+A e^{-B Q_{N o m}}-R i}{\left(Q_{N o m}+i\right)}\right)\left(\frac{Q-Q_{N o m}}{Q}\right),
$$

onde: $V_{\text {Nom }}$ é a tensão no final da zona nominal $(V)$, 


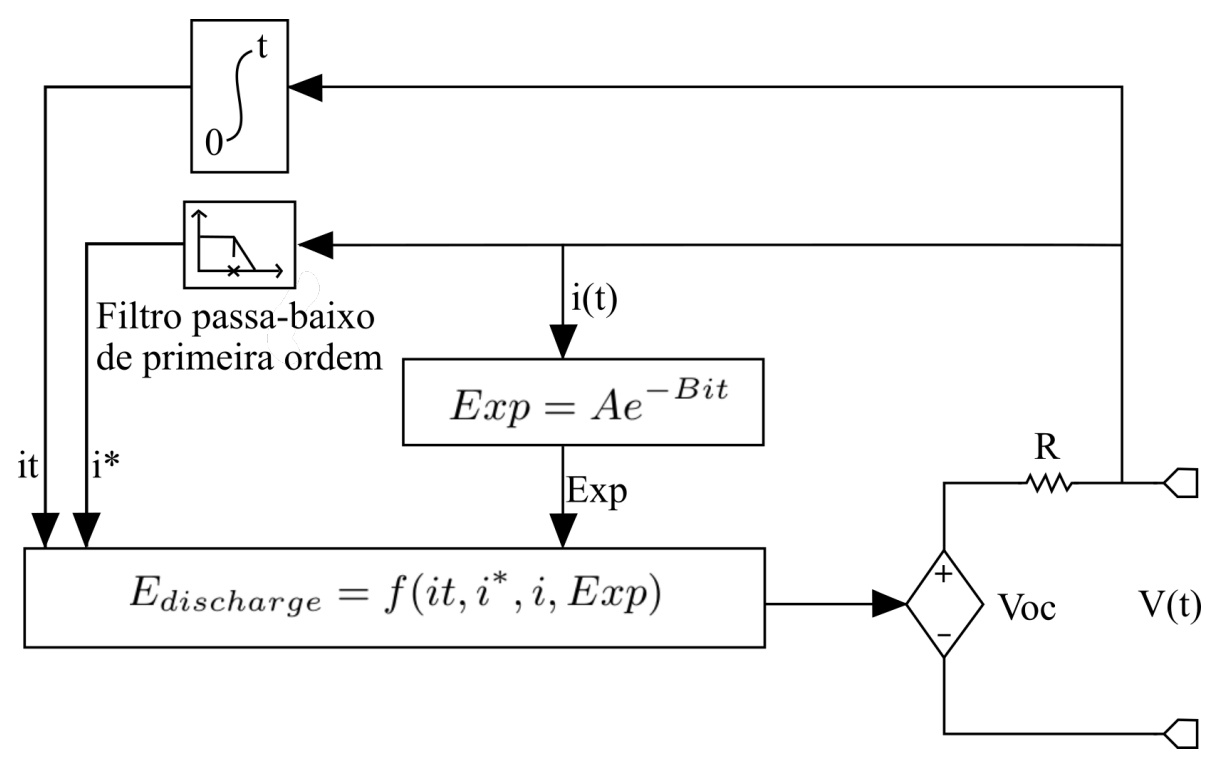

Figura 2: Circuito equivalente do modelo Battery (Brondani et al.; 2017b)

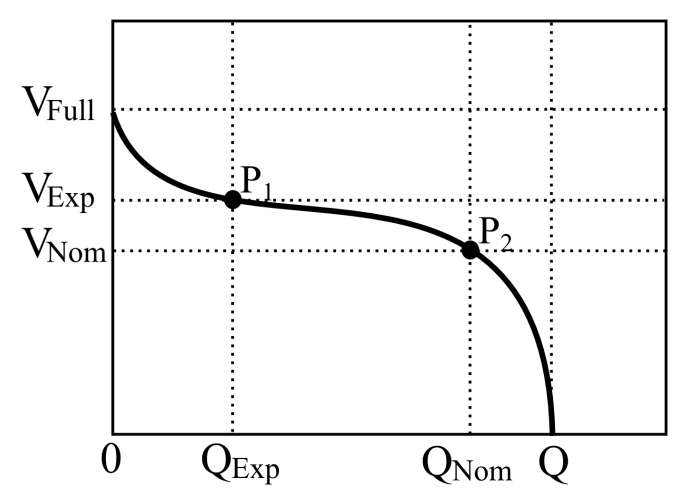

Figura 3: Ilustração de uma curva característica de descarga e dos parâmetros do modelo Battery

(Brondani et al.; 2017b)

e $Q_{\text {Nom }}$ é a capacidade no final da zona nominal (Ah). Em estado estacionário, a corrente filtrada $i^{*}$ é igual a $i$ (Tremblay and Dessaint; 2009).

Os parâmetros estimados neste trabalho, que dependem de dados obtidos em curvas de descarga, são: $V_{\text {Exp }}, Q_{E x p}, V_{\text {Nom }}$ e $Q_{\text {Nom. }}$. A metodologia usualmente adotada para a estimação desses parâmetros é baseada na análise visual dos pontos correspondentes a $P_{1}$ e $P_{2}$ em curvas reais de descarga de baterias, geradas com base em ensaios experimentais (Porciuncula et al.; 2015).

\section{Dados experimentais}

Nesta seção são apresentados os dados experimentais utilizados nos processos de parametrização e de validação dos modelos Chen e Rincón-Mora e Battery. Os dados são referentes a baterias de LiPo novas, modelo PL383562 - 2C, coletados em uma plataforma de testes desenvolvida pelo Grupo de Automação Industrial e Controle (GAIC), da Universidade Regional do Noroeste do Estado do Rio Grande do Sul (Unijuí).
Os testes experimentais realizados para a estimação dos parâmetros do modelo Chen e RincónMora são baseados no procedimento adotado por Chen and Rincón-Mora (2006), que utiliza quatro curvas de descargas pulsadas que são geradas a partir das informações contidas na Tabela 1, na qual são apresentadas as quatro correntes consideradas $(m A)$, os respectivos períodos ( $\min$ ) e a duração de cada pulso $(\mathrm{min})$. Os períodos são repetidos até as baterias atingirem a tensão de cutoff correspondente a $3 \mathrm{~V}$.

Tabela 1: Curvas de descarga pulsadas usadas para a estimação dos parâmetros do modelo Chen e Rincón-Mora.

\begin{tabular}{ccc}
\hline Corrente de descarga & Período & Duração do pulso \\
\hline 80 & 68,34 & 58,34 \\
160 & 40,00 & 30,00 \\
320 & 25,00 & 15,00 \\
640 & 17,50 & 7,50 \\
\hline
\end{tabular}

Conforme proposto em MathWorks (2017), a parametrização do modelo Battery é realizada a partir de uma única curva de descarga constante, que pode ser obtida no datasheet da bateria ou de forma experimental. Em Brondani et al. (2017b) foram considerados diferentes perfis de descargas constantes para a calibração desse modelo, que apresentou resultados mais precisos ao ser parametrizado com o perfil de $250 \mathrm{~mA}$, sendo utilizada a mesma metodologia de estimação de parâmetros aqui adotada. Desta forma, neste trabalho é utilizada uma curva de descarga constante de $250 \mathrm{~mA}$, sendo esta escolhida a partir de oito ensaios experimentais realizados para este perfil.

Para a validação dos modelos são considerados perfis de descargas constantes e variáveis, que abrangem correntes baixas, médias e altas, respeitando o limite máximo aceitável para as baterias aqui estudadas. Desta forma, primeiramente, são definidos 31 perfis de descargas constantes que 
Tabela 2: Perfis de descargas constantes utilizados para a validação dos modelos.

\begin{tabular}{cccccccccc}
\hline Perfis & $t_{v e 1}$ & $t_{v e 2}$ & $t_{v e 3}$ & $t_{v e 4}$ & $t_{v e 5}$ & $t_{v e 6}$ & $t_{v e 7}$ & $t_{v e 8}$ & $t_{v e m}$ \\
\hline 50 & 964,07 & 980,42 & 860,43 & 922,15 & 920,43 & 993,96 & 931,38 & 950,08 & 940,36 \\
75 & 593,06 & 607,28 & 626,98 & 599,75 & 586,9 & 652,15 & 582,58 & 606,78 & 606,93 \\
100 & 464,71 & 474,42 & 460,45 & 466,3 & 470,75 & 459,03 & 445,53 & 486,62 & 465,97 \\
125 & 388,48 & 392,93 & 375,03 & 367,4 & 380,9 & 393,65 & 381,55 & 398,17 & 384,76 \\
150 & 283,02 & 311,72 & 302,97 & 307,58 & 345,03 & 297,62 & 279,48 & 305,38 & 304,1 \\
175 & 280,85 & 274,5 & 271,33 & 285,07 & 297,07 & 253,5 & 246,43 & 269,1 & 272,23 \\
200 & 232,2 & 219,18 & 213,95 & 236,18 & 218,83 & 235,7 & 239,72 & 228,12 & 227,98 \\
325 & 141,57 & 145,68 & 149,53 & 143,47 & 148,45 & 129,85 & 126,4 & 145,33 & 141,28 \\
400 & 115,55 & 117,75 & 121,52 & 115,78 & 118,8 & 107,9 & 101,9 & 117,5 & 114,58 \\
525 & 88,57 & 91,3 & 93,15 & 85,28 & 92,63 & 78,67 & 74,11 & 85,85 & 86,19 \\
\hline
\end{tabular}

Tabela 3: Composição dos perfis de descargas variáveis.

\begin{tabular}{ccc}
\hline Perfis & Correntes do período & Duração de cada pulso \\
\hline$p_{1}$ & $100-10-150-10-100-10-200$ & $5-5-5-5-5-5-10$ \\
$p_{3}$ & $270-10-120-170-10-270-170$ & $5-10-10-15-10-15-5$ \\
$p_{4}$ & $250-400-50-200-550$ & $10-10-5-15-10$ \\
$p_{6}$ & $100-200-300-400-500-600-700$ & $10-10-10-10-10-10-10$ \\
$p_{7}$ & $700-600-500-400-300-200-100$ & $10-10-10-10-10-10-10$ \\
\hline
\end{tabular}

variam de $50 \mathrm{~mA}$ a $800 \mathrm{~mA}$, igualmente divididos em intervalos de $25 \mathrm{~mA}$. Para cada um desses perfis são realizados oito ensaios, obtendo o tempo de vida experimental médio $\left(t_{\text {vem }}\right)$ do perfil. Por meio de uma análise estatística utilizando One-Way ANOVA e Tukey, dentre esses perfis mensurados são selecionados 10 estatisticamente relevantes, mostrados na Tabela 2, com o tempo de vida experimental de cada ensaio $t_{v e i} \mathrm{em} \min$, onde $1 \leq i \leq 8$ e o $t_{\text {vem }}$ de cada perfil ( $\min$ ). As baterias são consideradas descarregadas quando alcançam a tensão de 2, $7 \mathrm{~V}$.

Em seguida, são determinados oito perfis de descargas variáveis baseando-se em valores de correntes medidos a partir da realização de atividades básicas em um telefone celular do tipo smartphone. Para cada um desses perfis também são realizados oito ensaios, e obtido o $t_{v e m}$ do perfil. Nessa nova base de dados são aplicados os mesmos testes estatísticos mencionados acima, resultando em cinco perfis significativamente diferentes, elencados na Tabela 3 , que contém as correntes de descarga $(m A)$ que compõem cada período e a duração de cada pulso (min). As baterias também são descarregadas até a tensão de $2,7 \mathrm{~V}$, sendo o tempo de vida experimental de cada ensaio $t_{v e i}$ em $\mathrm{min}$, onde $1 \leq i \leq 8$, e o $t_{v e m}$ de cada perfil $(\min )$, apresentados na Tabela 4 .

\section{Metodologias para estimação dos parâmetros}

Nesta seção são apresentadas as metodologias utilizadas na parametrização dos modelos, tendo como base a análise visual de curvas de descarga obtidas experimentalmente.

\subsection{Estimação dos parâmetros do modelo Chen e Rincón-Mora}

A estimação dos 21 parâmetros do modelo Chen e Rincón-Mora é fundamentada na técnica de ajuste de curvas e segue o procedimento descrito em Chen and Rincón-Mora (2006). Para cada uma das funções Voc, $R_{0}, R_{1}, C_{1}, R_{2}$ e $C_{2}$, apresentadas nas equações (6)-(8), são geradas quatro curvas experimentais, onde cada uma corresponde a um dos quatro perfis de descargas pulsadas apresentados na Tabela 1, ou seja, de $80 \mathrm{~mA}$, $160 \mathrm{~mA}, 320 \mathrm{~mA}$ e $640 \mathrm{~mA}$. Cada uma destas curvas contém um conjunto de pontos característicos que são obtidos dos pulsos das correntes de descarga. Neste contexto, o ajuste de curvas consiste em encontrar uma lei da função que passa por todos estes pontos, e por conseguinte, os respectivos parâmetros.

Assim, para determinar a função Voc, equação (6), é necessário medir a tensão em circuito aberto em diferentes pontos do SOC, que corresponde à tensão terminal de circuito aberto no estado estacionário em cada pulso de descarga (Chen and Rincón-Mora; 2006). A função $R_{0}$, equação (7), é determinada a partir do cálculo da resistência $R_{0}$ em cada um dos pulsos das correntes de descarga. Esta resistência é responsável pela queda de tensão instantânea no pulso, sendo consideradas as tensões inicial e final no instante em que é aplicada a corrente de descarga, conforme mostrado em Schweighofer et al. (2003). Para a obtenção das leis que definem as funções $R_{1}$ e $C_{1}$, apresentadas na equação (8), são calculados os valores de $R_{1}$ e $C_{1}$ considerando os primeiros segundos de cada um dos pulsos das correntes de descarga. Conforme descrito em Schweighofer et al. (2003), para o cálculo de $R_{1}$ é necessária a diferença de tensão no correspondente intervalo, e para o cálculo de $C_{1}$ é necessária a diferença de tempo, que é obtida traçando uma reta tangente ao ponto que corresponde à tensão incial do intervalo. E por fim, as funções $R_{2}$ and $C_{2}$, apresentadas na equação (8), dependem dos valores de $R_{2}$ and $C_{2}$, os quais são calculados considerando os segundos restantes de 
Tabela 4: Perfis de descargas variáveis utilizados para a validação dos modelos.

\begin{tabular}{cccccccccc}
\hline Perfis & $t_{v e 1}$ & $t_{\text {ve2 }}$ & $t_{\text {ve3 }}$ & $t_{\text {ve4 }}$ & $t_{\text {ve5 }}$ & $t_{\text {ve6 }}$ & $t_{\text {ve7 }}$ & $t_{\text {ve8 }}$ & $t_{\text {vem }}$ \\
\hline$p_{1}$ & 463,81 & 512,01 & 470,10 & 490,25 & 463,33 & 494,11 & 470,91 & 473,20 & 479,67 \\
$p_{3}$ & 340,23 & 330,50 & 308,86 & 313,81 & 304,16 & 333,60 & 331,00 & 303,93 & 322,01 \\
$p_{4}$ & 146,53 & 147,68 & 156,13 & 149,15 & 146,80 & 148,48 & 147,98 & 152,28 & 149,38 \\
$p_{6}$ & 135,49 & 133,50 & 125,56 & 124,68 & 124,23 & 122,68 & 120,00 & 126,79 & 126,62 \\
$p_{7}$ & 99,21 & 105,73 & 99,93 & 97,00 & 95,98 & 99,21 & 93,31 & 97,71 & 98,51 \\
\hline
\end{tabular}

cada um dos pulsos das correntes de descarga, e de maneira semelhante às constantes de curta duração (Schweighofer et al.; 2003).

Todas as medições necessárias são realizadas a partir da análise visual nos pontos específicos dos pulsos das correntes de descarga aplicadas às baterias. Após a obtenção do conjunto de dados correspondente a cada uma das funções, é utilizado o método dos Mínimos Quadrados Não Linear para o ajuste das curvas, implementado no software Matlab, conseguindo deste modo a equação que representa cada uma das funções do modelo Chen e RincónMora.

\subsection{Estimação dos parâmetros do modelo Battery}

Neste trabalho, 4 parâmetros do modelo Battery são estimados a partir da curva de descarga utilizada na parametrização, os quais são: $V_{E x p}, Q_{E x p}, V_{N o m} \mathrm{e}$ $Q_{\text {Nom }}$. A obtenção desses parâmetros, contidos nas equações (12)-(14), se dá por meio de uma curva de descarga constante que descreve o decaimento da tensão em função do tempo de execução da bateria. Assim, inicialmente, são geradas 8 curvas de descarga constantes correspondentes aos 8 experimentos independentes realizados para o perfil de $250 \mathrm{~mA}$. Em seguida, dentre essas curvas, seleciona-se aquela cujo tempo de vida se encontra mais próximo do $t_{\text {vem, }}$ conforme mostrado na Figura 4. Após a escolha da curva, seguindo a metodologia adotada em Porciuncula et al. (2015), esses parâmetros são estimados a partir da análise visual desta curva nos pontos equivalentes a $P_{1}$ e $P_{2}$, mostrados na Figura 3.

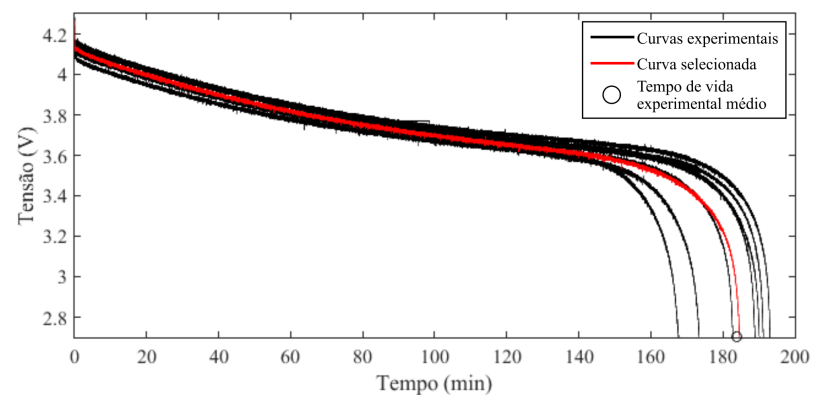

Figura 4: Curvas experimentais referentes ao perfil de descarga constante de $250 \mathrm{~mA}$

Os demais parâmetros do modelo são obtidos diretamente do datasheet da bateria e ajustados conforme proposto em MathWorks (2017). O SOC inicial é definido como $100 \%$, uma vez que no início do processo de descarga a bateria está totalmente carregada. A capacidade máxima é tida como $105 \%$ da capacidade nominal. O tempo de resposta é configurado para $30 \mathrm{~s}$, valor que representa satisfatoriamente a dinâmica da tensão da bateria.

\section{Resultados das simulações e análise}

Nesta seção são apresentados os resultados simulados, primeiramente, pelo modelo Chen e Rincón-Mora e, em seguida, pelo modelo Battery. Para cada modelo é apresentada a estimação dos parâmetros, e os modelos são validados de acordo com os 10 perfis de descargas constantes e os 5 perfis de descargas variáveis, apresentados respectivamente nas Tabelas 2 e 4. A análise dos resultados é realizada a partir da diferença entre os tempos de vida simulados e os tempos de vida experimentais, obtida por meio do cálculo do erro relativo percentual.

\subsection{Resultados simulados pelo modelo Chen e Rincón-Mora}

Inicialmente é apresentada a validação da estimação dos parâmetros do modelo Chen e Rincón-Mora a partir de simulações realizadas para os quatro perfis de descargas pulsadas utilizados no processo de parametrização, os quais são $80 \mathrm{~mA}, 160 \mathrm{~mA}, 320 \mathrm{~mA}$ e $640 \mathrm{~mA}$. Na Tabela 5 são apresentados os tempos de vida experimentais ( $t_{v e}$ em $\mathrm{min}$ ), os tempos de vida simulados ( $t_{v s}$ em $\mathrm{min}$ ), e os erros obtidos pelo modelo para esses perfis.

Tabela 5: Validação da estimação dos parâmetros do modelo Chen e Rincón-Mora.

\begin{tabular}{cccc}
\hline Perfis & $t_{v e}$ & $t_{v s}$ & Erro (\%) \\
\hline 80 & 655,50 & 672,67 & 2,62 \\
160 & 379,60 & 379,00 & 0,16 \\
320 & 227,84 & 232,67 & 2,12 \\
640 & 160,50 & 160,17 & 0,20 \\
\hline Erro médio (\%) & & & 1,28 \\
\hline
\end{tabular}

Para a validação da estimação, o modelo obteve um erro médio de $1,28 \%$, sendo considerado relativamente baixo, o que é esperado, uma vez que são utilizados no modelo os mesmos perfis de descarga do processo de parametrização. As curvas, simuladas e experimentais, referentes a esses quatro perfis podem ser vistas nas Figuras 5, 6, 7 e 8 . Nota-se que as curvas simuladas possuem um comportamento muito semelhante às curvas experimentais em praticamente todo intervalo de tempo da descarga, sendo observada uma pequena 
diferença nos pulsos das curvas correspondentes ao perfil de $320 \mathrm{~mA}$ (Figura 7), e também no fim das descargas para os perfis de $80 \mathrm{~mA}$ (Figura 6) e 320 $\mathrm{mA}$ (Figura 7), em que as curvas simuladas demoram um tempo maior para atingirem o nível de cutoff.

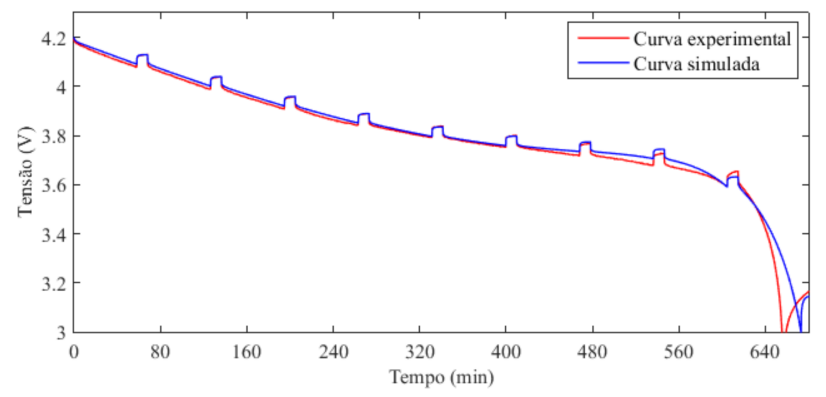

Figura 5: Curvas experimental e simulada considerando o perfil de $80 \mathrm{~mA}$

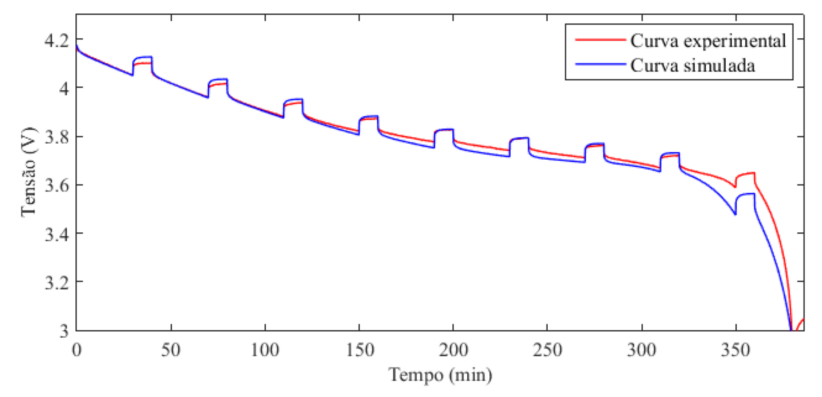

Figura 6: Curvas experimental e simulada considerando o perfil de $160 \mathrm{~mA}$

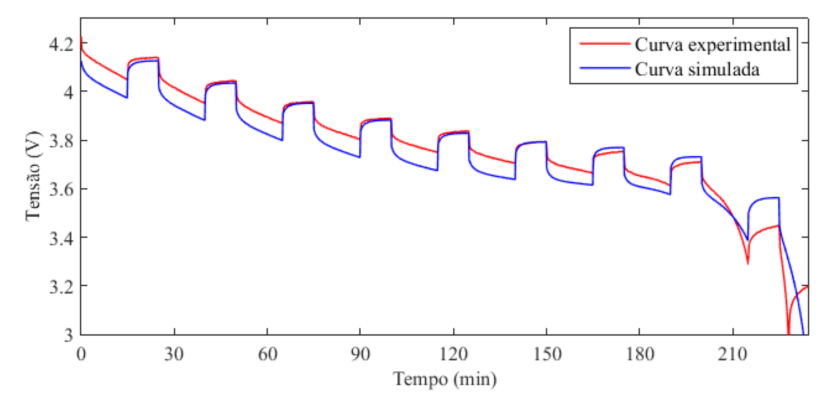

Figura 7: Curvas experimental e simulada considerando o perfil de $320 \mathrm{~mA}$

Os valores dos parâmetros estimados na parametrização do modelo Chen e Rincón-Mora são apresentados na Tabela 6 . A validação do modelo é realizada considerando 15 diferentes perfis de descarga que não foram utilizados no processo de parametrização, conforme descritos na Seção 3 (Tabelas 2 e 4). Nas Tabelas 7 e 8 são apresentados os resultados obtidos para os perfis de descargas constantes e variáveis, respectivamente. Em ambas tabelas podem ser vistos o $t_{v e m}(\mathrm{~min})$, o $t_{v s}(\mathrm{~min})$,

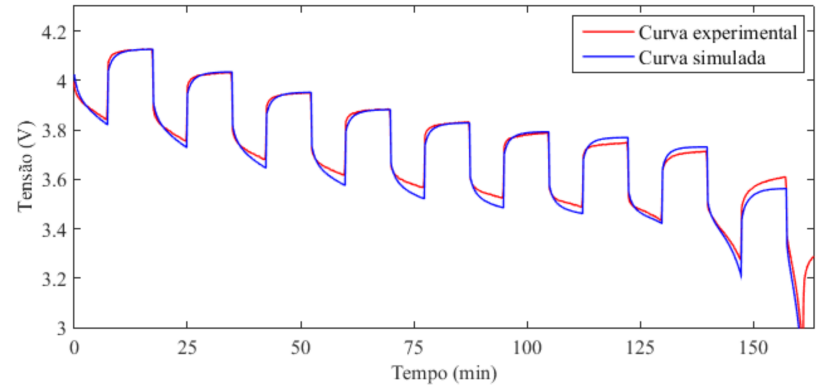

Figura 8: Curvas experimental e simulada considerando o perfil de $640 \mathrm{~mA}$

o erro correspondente a cada um dos perfis, e o erro médio obtido pelo modelo em cada uma das validações. Para essas simulações, considerando todos os perfis de descargas constantes e variáveis, 0 modelo obteve um erro médio geral de $1,79 \%$.

Tabela 6: Valores dos parâmetros do modelo Chen e Rincón-Mora.

\begin{tabular}{cc}
\hline Parâmetros & Valores \\
\hline$a_{0}$ & $-1,1275$ \\
$a_{1}$ & 13,0706 \\
$a_{2}$ & 3,9594 \\
$a_{3}$ & $-1,1079$ \\
$a_{4}$ & $-2,0267$ \\
$a_{5}$ & $-0,6548$ \\
$b_{0}$ & 3,0691 \\
$b_{1}$ & 64,1681 \\
$b_{2}$ & 0,3167 \\
$c_{0}$ & 18,1582 \\
$c_{1}$ & 151,13 \\
$c_{2}$ & 0,0706 \\
$d_{0}$ & $-534,1811$ \\
$d_{1}$ & 9,3313 \\
$d_{2}$ & 508,0335 \\
$e_{0}$ & 1,4902 \\
$e_{1}$ & 29,3493 \\
$e_{2}$ & 0,0971 \\
$f_{0}$ & $-1454,6938$ \\
$f_{1}$ & 8,5250 \\
$f_{2}$ & 1307,4889 \\
\hline
\end{tabular}

Tabela 7: Validação do modelo Chen e Rincón-Mora para perfis de descargas constantes.

\begin{tabular}{cccc}
\hline Perfis & $t_{v e m}$ & $t_{v s}$ & Erro (\%) \\
\hline 50 & 940,37 & 947,83 & 0,79 \\
75 & 606,93 & 630,00 & 3,80 \\
100 & 465,98 & 471,33 & 1,15 \\
125 & 384,77 & 376,17 & 2,24 \\
150 & 304,10 & 312,83 & 2,87 \\
175 & 272,23 & 267,67 & 1,68 \\
200 & 228,00 & 233,83 & 2,56 \\
325 & 141,28 & 142,75 & 1,04 \\
400 & 114,58 & 115,50 & 0,80 \\
525 & 86,20 & 87,42 & 1,41 \\
\hline Erro médio (\%) & & & 1,83 \\
\hline
\end{tabular}


Tabela 8: Validação do modelo Chen e Rincón-Mora para perfis de descargas variáveis.

\begin{tabular}{cccc}
\hline Perfis & $t_{v e m}$ & $t_{v s}$ & Erro (\%) \\
\hline$p_{1}$ & 479,67 & 479,70 & 0,01 \\
$p_{3}$ & 322,01 & 332,02 & 3,11 \\
$p_{4}$ & 149,38 & 152,93 & 2,38 \\
$p_{6}$ & 126,62 & 124,60 & 1,60 \\
$p_{7}$ & 98,51 & 99,93 & 1,44 \\
\hline Erro médio (\%) & & & 1,71 \\
\hline
\end{tabular}

\subsection{Resultados simulados pelo modelo Battery}

O modelo Battery é configurado com 4 dos seus parâmetros estimados mediante a análise visual de pontos específicos em uma curva de descarga constante de $250 \mathrm{~mA}$. Na Figura 9 são mostradas a curva experimental utilizada nesta parametrização e a curva simulada pelo modelo submetido a essa mesma corrente de descarga. Considerando a validação da estimação é possível observar que o comportamento da curva simulada assemelha-se ao da curva experimental, estando ambas muito próximas. Neste caso, o tve é de 184,63 min e o tvs é de $184,73 \mathrm{~min}$, obtendo o erro significativamente baixo de $0,05 \%$. Os valores dos parâmetros estimados são apresentados na Tabela 9.

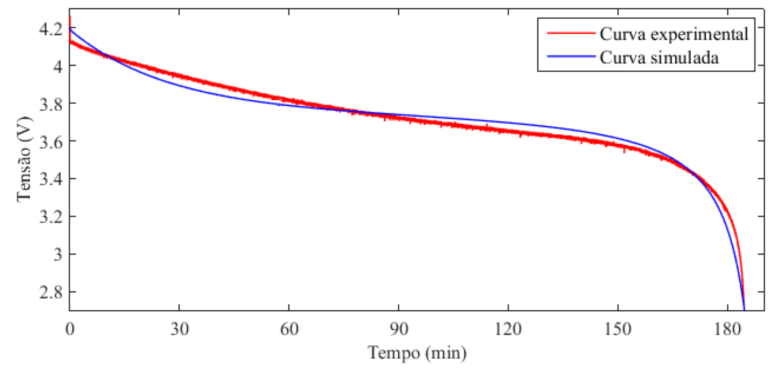

Figura 9: Validação da estimação dos parâmetros do modelo Battery

Tabela 9: Valores dos parâmetros do modelo Battery.

\begin{tabular}{cc}
\hline Parâmetros & Valores \\
\hline Tipo de bateria & LiPo \\
$V_{\text {Nom }}$ & $3,5 \mathrm{~V}$ \\
Capacidade nominal & $0,8 \mathrm{Ah}$ \\
SOC inicial & $100 \%$ \\
Capacidade máxima & $0,84 \mathrm{Ah}$ \\
$V_{\text {Full }}$ & $4,2 \mathrm{~V}$ \\
Corrente nominal de descarga & $0,25 \mathrm{~A}$ \\
$R$ & $0,028 \mathrm{Ohms}$ \\
$Q_{\text {Nom }}$ & $0,6897 \mathrm{Ah}$ \\
$V_{\text {Exp }}$ & $3,753 \mathrm{~V}$ \\
$Q_{\text {Exp }}$ & $0,3344 \mathrm{Ah}$ \\
Tempo de resposta & $30 \mathrm{~s}$ \\
Unidade & $\mathrm{s}$ \\
\hline
\end{tabular}

Os mesmos perfis de descarga utilizados para validar o primeiro modelo aqui apresentado, também são aplicados na validação do modelo Battery. Os resultados das simulações realizadas para os perfis de descargas constantes e variáveis são mostrados, respectivamente, nas Tabelas 10 e 11, as quais contêm o tvem ( $\mathrm{min}$ ), o tvs ( $\mathrm{min}$ ), o erro obtido pelo modelo para cada um dos perfis, e o erro médio para cada uma das validações. Considerando tais simulações, o modelo Battery obteve um erro médio geral de $1,37 \%$, i.e., para perfis de correntes constantes e variáveis.

Tabela 10: Validação do modelo Battery para perfis de descargas constantes.

\begin{tabular}{cccc}
\hline Perfis & $t_{v e m}$ & $t_{v s}$ & Erro $(\%)$ \\
\hline 50 & 940,37 & 930,60 & 1,04 \\
75 & 606,93 & 619,82 & 2,12 \\
100 & 465,98 & 464,45 & 0,33 \\
125 & 384,77 & 371,22 & 3,52 \\
150 & 304,10 & 309,05 & 1,63 \\
175 & 272,23 & 264,65 & 2,79 \\
200 & 227,98 & 231,35 & 1,48 \\
325 & 141,28 & 141,70 & 0,29 \\
400 & 114,58 & 114,80 & 0,19 \\
525 & 86,20 & 87,05 & 0,99 \\
\hline Erro médio (\%) & & & 1,44 \\
\hline
\end{tabular}

Tabela 11: Validação do modelo Battery para perfis de descargas variáveis.

\begin{tabular}{cccc}
\hline Perfis & $t_{v e m}$ & $t_{v s}$ & Erro (\%) \\
\hline$p_{1}$ & 479,67 & 477,35 & 0,48 \\
$p_{3}$ & 322,01 & 330,33 & 2,58 \\
$p_{4}$ & 149,38 & 149,82 & 0,29 \\
$p_{6}$ & 126,62 & 124,2 & 1,91 \\
$p_{7}$ & 98,51 & 99,43 & 0,93 \\
\hline Erro médio (\%) & & & 1,24 \\
\hline
\end{tabular}

\subsection{Análise comparativa entre os modelos}

Quando são comparados os resultados dos dois modelos, a maior diferença percebida é quanto ao erro do processo de obtenção dos parâmetros, sendo de $1,28 \%$ para o modelo Chen e Rincón-Mora, e de $0,05 \%$ para o modelo Battery. Isso deve-se principalmente ao fato de que para o modelo Chen e Rincón-Mora são utilizadas 4 diferentes curvas na sua parametrização, enquanto que para o modelo Battery é utilizada apenas 1, sendo assim mais fácil ajustar os dados a partir de uma única curva. Outro fator importante é a quantidade de parâmetros, para o modelo Chen e Rincón-Mora é necessária a estimação de 21 parâmetros, já para o modelo Battery é feita a estimação de apenas 4.

$\mathrm{Na}$ validação dos modelos, quando são considerados todos os perfis de descargas constantes e variáveis, o modelo Battery também apresenta um melhor desempenho, com um erro médio geral de $1,37 \%$, enquanto que o erro médio geral 
apresentado pelo modelo Chen e Rincón-Mora é de $1,79 \%$. De qualquer forma, ambos resultados são bons, considerando que o limite aceitável de erro para modelos elétricos é de até $5 \%$ (Chen and Rincón-Mora; 2006).

\section{Conclusão}

Neste trabalho foi apresentada a modelagem matemática do tempo de vida de baterias de LiPo por meio dos modelos Chen e Rincón-Mora e Battery. Apresentou-se o processo de parametrização, e fezse a validação dos modelos pela comparação entre os dados simulados e os dados experimentais obtidos em uma plataforma de testes. A partir da análise dos resultados é possivel verificar que ambos modelos são acurados, apresentando erros médios inferiores a $2 \%$. No entanto, o modelo Battery apresenta melhor desempenho geral associado a possuir um processo de parametrização mais simples quando comparado com o modelo Chen e Rincón-Mora. Como trabalhos futuros pretende-se utilizar outras tecnologias de baterias, assim como aplicar metaheurísticas para realizar a estimação de parâmetros de modelos elétricos.

\section{Agradecimentos}

À Coordenação de Aperfeiçoamento de Pessoal de Nível Superior (Capes) pelo incentivo financeiro, à Universidade Regional do Estado do Rio Grande do Sul (Unijuí) pela estrutura disponibilizada, e ao Grupo de Automação Industrial e Controle (Gaic), em especial, a Eduardo Cardoso Toniazzo, bolsista de iniciação científica, pela realização dos experimentos na plataforma de testes.

\section{Referências}

Binelo, M. F. B., Motyczka, L. B., Sausen, A. T. Z. R., Sausen, P. S. and Binelo, M. O. (2017). Battery Charge and Discharge Behavior Prediction Using Electrical Mathematical Models, Advances in Mathematics Research, Nova Science Publishers Incorporated.

Brand, J., Zhang, Z. and Agarwal, R. K. (2014). Extraction of battery parameters of the equivalent circuit model using a multi-objective genetic algorithm, Journal of Power Sources 247: 729 - 737.

Brondani, M. F., Sausen, A., Sausen, P. S. and Binelo, M. O. (2017a). Battery model parameters estimation using simulated annealing, TEMA - Tendências em Matemática Aplicada e Computacional 18: 127-135.

Brondani, M. F., Sausen, A. T. Z. R., Sausen, P. S. and Binelo, M. O. (2017b). Parameter estimation of lithium ion polymer battery mathematical model using genetic algorithm, Computational and Applied Mathematics pp. 1-18.

Chen, M. and Rincón-Mora, G. (2006). Accurate electrical battery model capable of predicting runtime and $\mathrm{i}-\mathrm{v}$ performance, IEEE Transactions on Energy Conversion 21(2): 504-511.
Chiasserini, C. and Rao, R. (1999). Pulsed battery discharge in communication devices, Proceedings of the 5th International Conference on Mobile Computing and Networking pp. 88-95.

Doyle, M., Fuller, T. F. and Newman, J. S. (1993). Modeling of galvanostatic charge and discharge of the lithium/polymer/insertion cell, Journal of The Electrochemical Society 140: 1526-1533.

Fotouhi, A., Auger, D. J., Propp, K., Longo, S. and Wild, M. (2016). A review on electric vehicle battery modelling: From lithium-ion toward lithium-sulphur, Renewable and Sustainable Energy Reviews 56: 1008 - 1021.

Hammond, G. P. and Hazeldine, T. (2015). Indicative energy technology assessment of advanced rechargeable batteries, Applied Energy 138: 559 571.

He, H., Xiong, R., Guo, H. and Li, S. (2012). Comparison study on the battery models used for the energy management of batteries in electric vehicles, Energy Conversion and Management 64: 113 - 121. \{IREC\} 2011, The International Renewable Energy Congress.

He, H., Xiong, R., Zhang, X., Sun, F. and Fan, J. (2011). State-of-charge estimation of the lithiumion battery using an adaptive extended kalman filter based on an improved thevenin model, IEEE Transactions on Vehicular Technology 60(4): 14611469.

$\mathrm{Hu}$, T. and Jung, H. (2013). Simple algorithms for determining parameters of circuit models for charging/discharging batteries, Journal of Power Sources 233: $14-22$.

IEA, I. E. A. (2017). Global ev outlook 2017. Disponível em https://www.iea.org/ publications/freepublications/publication/ GlobalEVOutlook2017.pdf (Acessado 6 Jan. 2018).

ITU, I. T. U. (2017). Measuring the information society report 2017. Disponível em https: //www.itu.int/en/ITU-D/Statistics/Documents/ publications/misr2017/MISR2017_Volume1.pdf (Acessado 28 Dez. 2017).

Kim, T. and Qiao, W. (2011). A hybrid battery model capable of capturing dynamic circuit characteristics an nonlinear capacity effects, IEEE Wireless Communications and Networking Conference 26: $1172-1180$.

Li, S. E., Wang, B., Peng, H. and Hu, X. (2014). An electrochemistry-based impedance model for lithium-ion batteries, Journal of Power Sources 258: 9 - 18 .

Lin, C., Mu, H., Xiong, R. and Shen, W. (2016). A novel multi-model probability battery state of charge estimation approach for electric vehicles using $\mathrm{h}-$ infinity algorithm, Applied Energy 166: 76 - 83 .

Luo, T., Li, L., Ghorband, V., Zhan, Y., Song, H. and Christen, J. B. (2016). A portable impedancebased electrochemical measurement device, 2016 IEEE International Symposium on Circuits and Systems (ISCAS), pp. 2891-2894. 
Manzetti, S. and Mariasiu, F. (2015). Electric vehicle battery technologies: From present state to future systems, Renewable and Sustainable Energy Reviews 51: $1004-1012$.

MathWorks (2017). Implement generic battery model. Disponível em https://www . mathworks.com/ help/physmod/sps/powersys/ref/battery.html?s_ tid=srchtitle (Acessado 10 Nov. 2017).

Mousavi, S. and Nikdel, M. (2014). Various battery models for various simulation studies and applications, Renewable and Sustainable Energy Reviews 32: 477 - 485 .

Panigrahi T, D., Panigrahi, D., Chiasserini, C., Dey, S., Rao, R., Raghunathan, A. and Lahiri, K. (2001). Battery life estimation of mobile embedded systems, VLSI Design, 2001. Fourteenth International Conference on, pp. 57-63.

Pattipati, B. R., Sankavaram, C. and Pattipati, K. R. (2011). System identification and estimation framework for pivotal automotive battery management system characteristics, IEEE Trans. Systems, Man, and Cybernetics, Part C 41(6): 869-884.

Porciuncula, C. M. D., Sausen, A. T. Z. R. and Sausen, P. S. (2015). Mathematical Modeling for Predicting Battery Lifetime through Electrical Models, Advances in Mathematics Research, Nova Science Publishers Incorporated.

Rakhmatov, D. and Vrudhula, S. (2001). An analytical high-level battery model for use in energy management of portable electronic systems, National Science Foundation's State/Industry/University Cooperative Research Centers (NSFS/IUCRC) Center for Low Power Electronics (CLPE) pp. 1-6.

Rezvanizaniani, S. M., Liu, Z., Chen, Y. and Lee, J. (2014). Review and recent advances in battery health monitoring and prognostics technologies for electric vehicle (ev) safety and mobility, Journal of Power Sources 256: 110 - 124.

Romio, L. C., Sausen, A. T. Z. R., Sausen, P. S. and Reimbold, M. (2015). Mathematical Modeling of the Lithium-ion battery Lifetime using System Identification Theory, Advances in Mathematics Research, Nova Science Publishers Incorporated.

Sausen, P. S., Spohn, M. A. and Perkusich, A. (2010). Broadcast routing in wireless sensor networks with dynamic power management and multi-coverage backbones, Information Sciences 180(5): 653 - 663.

Schweighofer, B., Raab, K. M. and Brasseur, G. (2003). Modeling of high power automotive batteries by the use of an automated test system, IEEE Transactions on Instrumentation and Measurement 52(4): 10871091.

Seaman, A., Dao, T.-S. and McPhee, J. (2014). A survey of mathematics-based equivalent-circuit and electrochemical battery models for hybrid and electric vehicle simulation, Journal of Power Sources 256: $410-423$.
Suthar, B., Ramadesigan, V., Northrop, P. W. C., Gopaluni, R. B., Santhanagopalan, S., Braatz, R. D. and Subramanian, V. R. (2013). Optimal control and state estimation of lithium-ion batteries using reformulated models, American Control Conference, ACC 2013, Washington, DC, USA, June 17-19, 2013, pp. 5350-5355.

Tremblay, O. and Dessaint, L.-A. (2009). Experimental validation of a battery dynamic model for ev applications, World Electric Vehicle Journal 3: 289-298.

Tremblay, O., Dessaint, L.-A. and Dekkiche, A.-I. (2007). A generic battery model for the dynamic simulation of hybrid electric vehicles, Vehicle Power and Propulsion Conference, 2007. VPPC 2007. IEEE, pp. 284-289.

Zhang, C., Li, K., Mcloone, S. and Yang, Z. (2014). Battery modelling methods for electric vehicles a review, 2014 European Control Conference (ECC), pp. 2673-2678. 